BIBLID: 0015-1807, 47 (2020), 2 (pp. 139-148

UDC 811.133.1'374:59(031)=133.1"12"

https://doi.org/10.18485/fpregled.2020.47.2.10

\author{
Marija Panić \\ Université de Kragujevac \\ ms.marija.panic@gmail.com
}

\title{
LA ZOOLOGIE DANS L'IMAGE DU MONDE DE GOSSOUIN DE METZ
}

Résumé : Le présent article traite de la représentation du monde animal dans l'encyclopédie l'Image du monde de Gossouin de Metz (XIII' siècle). Nous analysons les sources que l'encyclopédiste messin a utilisées, puis sa classification des animaux et leur représentation dans ce texte. En comparant son ouvrage avec d'autres textes didactiques de son époque (zoologiques et cosmographiques), nous cherchons à établir l'importance de la dimension symbolique de cet ouvrage.

Mots-clés : Gossouin de Metz, Image du monde, animal, symbolisme, encyclopédisme médiéval.

\begin{abstract}
This paper examines the representation of animals in the encyclopedia Image du monde, written by Gossouin de Metz in the $13^{\text {th }}$ century, especially the sources used by Gossouin de Metz, the classification of animals, as well as their representation in this text. The aim is to establish the importance of symbolism in the Image du monde, by comparing this encyclopedia with other medieval texts on zoology and cosmography.
\end{abstract}

Keywords: Gossouin de Metz, Image du monde, animal, symbolism, Medieval encyclopedism.

Le symbolisme était un trait incontournable dans les textes médiévaux sur la nature : il fallait non seulement représenter un fait sur le monde animal ou sur la géographie, mais aussi l'interpréter de façon élaborée, de sorte à édifier le lecteur. Or, cette dimension symbolique a diminué au cours du XIII ${ }^{\mathrm{e}}$ siècle, ce qui est le résultat de la traduction des textes d'Aristote sur la nature, qui n'avaient pas d'interprétation symbolique. Le présent article traite de la représentation du monde animal dans l'Image du monde de Gossouin de Metz, première encyclopédie rédigée en langue vulgaire, à l'époque où l'influence du nouveau naturalisme était déjà visible.

\section{L'Image du monde de Gossouin de Metz}

L'Image du monde de Gossouin de Metz, rédigé au milieu du XIII ${ }^{\mathrm{e}}$ siècle $^{1}$, est un ouvrage de vulgarisation scientifique qui a connu une grande notoriété dès

${ }^{1}$ Gossouin de Metz a composé la première version de l'Image du monde en 1246 ; il s'agit de la première version en vers, qui comporte plus de six mille octosyllabes. La rédaction de la deuxième 
sa parution et dans les époques qui ont suivi. Il s'agit de la première somme de connaissances rédigée en langue vulgaire : ce texte est le résultat d'une compilation et non pas d'une simple traduction ou d'un remaniement d'un texte latin. Ce texte a connu trois rédactions. C'est la version en prose qui a connu le plus grand succès à son époque; elle a été traduite en plusieurs langues. En plus, c'est l'Image du monde de Gossouin de Metz, version en prose, qui a été le premier ouvrage illustré imprimé en Angleterre, en 1480 ; ceci témoigne de la longévité de la popularité de ce texte ${ }^{2}$.

Cette première encyclopédie en langue vulgaire compte parmi les premiers ouvrages inspirés par le nouvel esprit naturaliste, né au premier tiers du XIII ${ }^{\mathrm{e}}$ siècle. En particulier, à la différence des ouvrages des siècles précédents, qui représentaient la nature comme un ensemble de signes menant aux vérités divines, c'est-à-dire, comme comme c'était le cas du « Livre de la nature », au temps de l'encyclopédiste messin l'allégorie quittait lentement les textes sur la nature. Les animaux n'étaient plus vus comme un point de départ pour une interprétation symbolique explicite ; les auteurs, dont quelques-uns étaient dotés d'une véritable curiosité scientifique ${ }^{3}$, avaient pour objectif de décrire le monde plutôt que de l'expliquer comme un ensemble de symboles ${ }^{4}$. Il s'agit, bien sûr, de connaissances zoologiques encore très frustes, obtenues par le biais de sources livresques : l'Image du monde pullule d'animaux fabuleux (que nous évoquerons plus tard dans l'article), tout comme les autres textes du Moyen Âge.

\section{La zoologie médiévale : classification des espèces, sources, symbolisme}

Dans la zoologie médiévale, la division du monde animal était fondée sur la tradition biblique - selon le jour de la création (le cinquième jour pour les poissons et les animaux, le sixième pour les bêtes) - et la tradition antique, en fonction

version en vers a lieu l'année suivante, en 1247. Cette version, largement remaniée, comporte plus de dix mille vers (Chantal Connochie, « Gossuin de Metz », dans Claude Gauvard, Alain de Libera et Michel Zink (dir.), Dictionnaire du Moyen Âge, Paris, PUF, 2002, p. 597). La rédaction en prose, que nous avons utilisée pour notre article, a été composée sur le fondement de la première version en vers, avant la deuxième version en vers (Oscar H.Prior [éd.], L'Image du monde du maître Gossouin : rédaction en prose, Lausanne, Librairie Payot et Cie, 1913, pp. 8-11).

${ }^{2}$ Pour le présent article, nous avons employé la version en prose, éditée par Oscar Prior en 1913.

${ }^{3}$ Voir, à titre d'exemple, Robert Delort, Les animaux ont une histoire, Paris, Seuil, 1984, pp. 60-62; Frančesko Zambon, „Teologija bestijarijuma”, (prevela Aleksandra Mančić Milić), Gradac n75-78, 1986-1987, str. 121-134; Umberto Eko, Umetnost i lepo u estetici srednjeg veka (prevele Tanja Majstorović i Snežana Brajović), Novi Sad, Svetovi / Beograd, Kultura, 1992, p. 120.

${ }^{4}$ Sur la dimension symbolique de la zoologie médiévale on pourra consulter : Xénia Muratova, «Adam donne leurs noms aux animaux. L'iconographie de la scène dans l'art du Moyen Âge : les manuscrits des bestiaires enluminés du XII e et du XIII ${ }^{\mathrm{e}}$ siècle » Studi medievali Firenze n ${ }^{\circ}$ 8/2, 1977, pp. 367-394; Tullio Gregory, « Nature », dans Jacques Le Goff, Jean-Claude Schmitt et Jean Alessio (éd.), Dictionnaire raisonné de l'Occident médiéval, Paris, Fayard, 1999, pp. 806-819; Marija Panić, „Jedan vid srednjovekovne zoomorfne simbolike: prikaz i tumačenje prirode u francuskim bestijarima”, Zbornik Matice srpske za književnost i jezik n63/III, 2015, str. 695-718. 
du milieu où les animaux vivent et de la manière de laquelle ils se déplacent. La classification la plus répandue était celle qui divisait les animaux en bêtes, quadrupèdes terrestres qui se déplacent en marchant; oiseaux, qui habitent les airs et qui volent ; reptiles, qui rampent sur la terre ; poissons, qui nagent dans l'eau. Les classifications variaient selon l'auteur. À titre d'exemple, Isidore de Séville, célèbre encyclopédiste médiéval qui a exercé une grande influence sur tous les domaines des connaissances médiévales, divisait, dans le XII ${ }^{\mathrm{e}}$ livre de ses Etymologies, les animaux en huit catégories : 1) le bétail et les bêtes de somme ; 2) les bêtes sauvages ; 3) les petits animaux ; 4) les serpents ; 5) les vers ; 6) les poissons ; 7) les oiseaux ; 8) les petits animaux ailés ${ }^{5}$.

Les textes zoologiques médiévaux étaient composés à la base d'un nombre réduit de textes provenant de l'Antiquité. Pline l'Ancien et Solin sont les auteurs qui ont procuré à la zoologie médiévale des exemples les plus étonnants. L'ouvrage Historia naturalis de Pline l'Ancien, encyclopédiste du Ir siècle, comporte trente-sept volumes ; les livres VIII-XI sont consacrés aux animaux, c'est-à-dire aux bêtes, poissons, oiseaux et insectes (en outre, les livres XXVIII-XXX et XXXII décrivent des remèdes tirés des animaux). Cette vaste encyclopédie est rédigée par la méthode de la compilation : Pline a puisé les données zoologiques dans des sources antiques variées et cité des auteurs antiques qui lui ont fourni cette matière si abondante et extraordinaire. En fait, sans grand souci critique, Pline a transmis des connaissances variées, parfois contradictoires, sur les faits naturalistes qu'il développait dans son encyclopédie.

C'est surtout l'ouvrage de Solin, Collectanea rerum memorabilum, composé au III ${ }^{\mathrm{e}}$ siècle, qui a fait diffuser au Moyen Âge de nombreuses merveilles (mirabilia), ou monstres - peuples et créatures étranges - qui hantaient l'imaginaire médiéval. En effet, les créatures étranges et bizarres à cette époque intéressaient les lecteurs plus que la réalité environnante. Les auteurs chrétiens surtout profitaient de l'étrangeté de ces descriptions zoologiques pour transmettre leur message édifiant : notamment, plus un exemple était extraordinaire, plus puissante était sa vertu persuasive.

Un autre savant a fourni à l'époque médiévale des connaissances qui apparaissaient régulièrement dans les textes sur la nature. Il s'agit d'Isidore de Séville, dont l'influence sur la zoologie médiévale a été sans aucun doute prépondérante, surtout grâce à sa méthode étymologique ${ }^{6}$. Son encyclopédie Etymologie

${ }^{5}$ Les huit parties du XII ${ }^{\mathrm{e}}$ livre des Etymologies sont intitulées en concordance avec cette division du monde animal : De pecoribus et iumentis, De bestiis, De minutis animantibus, De serpentibus, De vermibus, De piscibus, De avibus, De minutis volatilibus.

${ }^{6}$ Nous employons ce terme dans l'acception de l'étymologie médiévale, c'est-à-dire la recherche de la vérité, du sens caché, dans le langage, par le rapprochement des mots qui ont une expression semblable. L'étymologie médiévale supposait qu'il était possible de connaître l'essence des choses par leur nom. Sur l'étymologie médiévale on peut consulter : Michel Pastoureau, Une histoire symbolique du Moyen Âge occidental, Paris, Seuil, 2004, pp. 14-17 ; Armand Strubel, 'Grant sénéfiance a': allégorie et littérature au Moyen Âge, Paris, Honoré Champion, 2002, pp. 80-81. 
(Etymologiae sive Originum, $\mathrm{VII}^{\mathrm{e}}$ siècle) compte parmi les ouvrages les plus diffusés au Moyen Âge. Dans ce texte, l'évêque wisigothique a systématisé les connaissances issues des textes antiques, en y ajoutant les pensées des Pères de l'Eglise et une interprétation chrétienne ${ }^{7}$. Son ouvrage comporte vingt livres, dont le XII , intitulé De animalibus, est consacré à la zoologie. Selon l'étymologie isidorienne, l'essence d'une chose est contenue dans son nom ; une ressemblance sonore entre deux mots signifie qu'il existe un lien ontologique qui rapproche les deux notions ${ }^{8}$. Pour ainsi dire, chaque description des animaux chez Isidore de Séville contient des explications étymologiques, qui étaient reprises dans les encyclopédies et dans les bestiaires médiévaux.

Un autre auteur antique a largement influencé les connaissances médiévales sur la nature. Il s'agit d'Aristote, dont les œuvres ont été traduits au XIII ${ }^{\mathrm{e}}$ siècle et ont enrichi, à partir de ce moment-là, les connaissances médiévales sur la nature. En effet, à l'époque de la création du Physiologus, ancêtre gréco-chrétien des bestiaires médiévaux, Aristote n'était pas utilisé comme source : le bizarre et l'extraordinaire étaient attendus dans la littérature naturaliste de l'époque hellénistique'. C'est pourquoi les mirabilia ont envahi l'imaginaire médiéval. Les textes d'Aristote Histoire des animaux, De la génération des animaux, Des parties des animaux, Du mouvement des animaux, étaient traduits en 1230 de l'arabe en latin par Michel Scot, et en 1260 du grec en latin par Guillaume de Marboecke. Ces écrits divisaient les animaux selon la morphologie de leurs organes et selon leurs fonctions : c'était la première fois que dans la zoologie médiévale apparaissait une approche rationnelle et systématique. Dans les textes aristotéliciens et dans les commentaires arabes qui les accompagnaient, il n'y avait pas d'interprétations édifiantes. Ceci est une grande innovation par rapport aux textes zoologiques médiévaux : dans la zoologie médiévale notamment, les descriptions des animaux servaient comme points de départ pour donner une édification chrétienne. Aristote était employé comme source déjà dans la deuxième moitié du XIII ${ }^{\mathrm{e}}$ siècle, dans les grandes encyclopédies de Vincent de Beauvais, de Barthélemy l'Anglais, d'Albert le Grand, et d'autres.

Le premier manuel zoologique que l'on peut considérer comme moderne date du XVI ${ }^{\mathrm{e}}$ siècle. Il s'agit de l'ouvrage Historiae animalium de Konrad Gessner,

${ }^{7}$ Étant donné qu'il a systématisé et classifié les connaissances médiévales et inclus celles des auteurs antiques, Isidore de Séville est vénéré dans le monde catholique comme saint patron d'Internet.

${ }^{8}$ À titre d'exemple, selon Isidore de Séville, l'aigle (aquila) se nomme ainsi grâce à l'acuité de ses yeux (acumen oculorum) ; une abeille (apis) porte ce nom parce qu'elle n'a pas de pieds (a-pes), et ainsi de suite. Les Etymologies d'Isidore de Séville ont connu peu de traductions en langues vulgaires, malgré leur popularité. En fait, c'est cette omniprésence des développements étymologiques (qui perdraient leur capacité de persuasion dans la traduction en langue vulgaire) qui a empêché les clercs médiévaux de traduire ce texte en langues vulgaires (Duval, Frédéric, « Les traductions françaises d'Isidore de Séville au Moyen Âge ", Cahiers de recherches médiévales et humanistes $\mathrm{n}^{\circ} 16,2008$, p. 93).

${ }^{9}$ Dragoljub Dragojlović, Fiziolog $u$ Srba (thèse de doctorat), Faculté de philosophie, Université de Belgrade, Univerzitetska biblioteka à Belgrade, cote RD 3451, 1968, pp. 3-5. 
rédigé entre 1551 et 1558 . Avant cette époque, la zoologie était fantaisiste, fabuleuse, et représentait les animaux imaginaires - licornes, griffons et d'autres - autant que les animaux familiers : tourterelles, loups, renards, belettes. Les descriptions de ces derniers avaient elles aussi peu de rapports avec la réalité. Un autre trait significatif de la zoologie médiévale était de donner les animaux en exemple afin de transmettre aux lecteurs un message édifiant. Cette pratique d'allégorisation des animaux était très répandue au Moyen Âge : avant le XIII siècle et la traduction des textes arabes sur la zoologie d'Aristote, l'histoire naturelle servait surtout à l'édification. La pratique d'interpréter symboliquement les descriptions des animaux disparaissait lentement au cours du XIII ${ }^{\mathrm{e}}$ siècle et le premier ouvrage sur la nature dépourvu d'allégorie est le Compendium philosophie, datant du début du XIV siècle.

En somme, les descriptions zoologiques médiévales se caractérisent par le manque de rapport à la réalité environnante, par leur dépendance des sources écrites et par l'absence d'une systématisation stricte. Quelques animaux étaient décrits en fonction des qualités de la matière (chaud/froid, humide/sec), dont se servait aussi la médecine médiévale. À titre d'exemple, la salamandre, vu son caractère froid, était dotée de la capacité d'éteindre le feu ${ }^{10}$; le bouc était considéré être un animal chaud, dont le sang peut briser un diamant ${ }^{11}$.

Il faut souligner que les descriptions zoologiques n'exposaient pas méthodiquement ni systématiquement les données que nous venons d'énumérer ; la plupart des textes zoologiques contenaient seulement des descriptions étonnantes de l'aspect physique ou du comportement de l'animal ${ }^{12}$.

\section{La zoologie dans l'Image du monde}

Le texte didactique de Gossouin de Metz est composé de trois parties. La première partie, contenant quatorze chapitres, traite de la cosmogonie, de Dieu, des sept arts libéraux, de la forme de l'univers et la terre, de quatre éléments. La deuxième partie, composée de dix-neuf chapitres, est consacrée à la géographie, c'est-à-dire aux points cardinaux et aux trois continents connus à l'époque : à l'Asie, à l'Europe et à l'Afrique. La troisième partie, contenant vingt-deux chapitres, est dédiée à l'astronomie et aux calculs des dimensions de la Terre.

Pour rédiger son ouvrage didactique, l'encyclopédiste messin a employé comme sources celles que nous avons déjà citées, y compris les ouvrages latins

\footnotetext{
${ }^{10}$ Michel Pastoureau, Les bestiaires du Moyen Âge, Paris, Seuil, 2011, p. 209.

${ }^{11}$ Ibid., p. 116.

${ }^{12}$ À titre d'exemple, voici la synthèse de quelques descriptions zoologiques du paon : il était décrit comme un animal au plumage d'une beauté exceptionnelle, mais qui a une grande crainte d'observer ses pieds. S'il se réveille la nuit, il crie de peur d'avoir perdu sa beauté ; cet animal était l'emblème de la vanité. Sa queue a beaucoup d'yeux ; c'est pourquoi il est interprété comme symbole de la sagesse, de la prudence (Edward B. Ham, [éd.], « The Cambrai Bestiary », Modern Philology n³6/3, 1939, p. 23 ; Craig Alexander Baker [éd.], Le Bestiaire, version longue attribuée à Pierre de Beauvais, Paris, Honoré Champion, 2010, pp. 164-165 ; Michel Pastoureau, op. cit., 2011, p. 166).
} 
populaires et très diffusés à son époque : Imago mundi de Honorius Augustodunensis, encyclopédie rédigée par la méthode de compilation, composée au XI ${ }^{\mathrm{e}}$ siècle; De Naturis rerum d'Alexandre Neckam, somme des connaissances datant du début du XIII ${ }^{\mathrm{e}}$ siècle ; Otia imperialia (Les divertissements pour un empereur) de Gervaise de Tilbury, ouvrage encyclopédique destiné à Henri le Jeune, prince d'Angleterre, puis à l'empereur Otton $\mathrm{IV}^{13}$; Historia orientalis (Histoire orientale), composée par Jacques de Vitry entre 1216 et 1227, lorsqu'il était évêque à Saint-Jean d'Acre en Terre sainte. Toutes ces encyclopédies jouissaient d'une popularité incontestée dans leurs époques.

La zoologie est contenue dans la deuxième partie de l'encyclopédie, dans les chapitres décrivant les continents (Asie, Europe, Afrique). Les descriptions de l'Asie étaient concentrées surtout sur l'Inde, qui était décrite au Moyen Âge comme un espace peuplé de mirabilia ${ }^{14}$. Certains chapitres sont consacrés exclusivement aux animaux («Des serpenz et des bestes d'Ynde », ii D; «Des poissons d'Ynde », ii G ; « De la maniere des bestes », vi B ; « De la maniere des oisiaus », vi C) ; dans les autres chapitres, apparaissent des données sur les animaux, parsemées à différents endroits du texte : « D'Ynde et de ses choses » (ii B), « Des diversitez d'Ynde » (ii C) ; «Des contrées d'Ynde » (ii F) ; « Des diversitez qui sont en Europe et en Aufrique » (vi A).

Dans le Tableau ci-dessous, nous citons une liste d'animaux, qui n'est pas exhaustive, puisque quelques animaux hybrides ont été décrits sans être explicitement nommés.

\section{Liste des animaux dans l'Image du monde de Gossouin de Metz}

\begin{tabular}{|l|l|}
\hline Asie (Inde) & Europe, Afrique \\
\hline dragons, griffons sauvages (ii B), grues, ver- & renard, cerf, araignée, lynx, singe, chien, \\
mine (ii C); serpents, centicore, manticora, & hérisson, belette (vi B); aigle, tourterelle, \\
bœufs à trois cornes, taureau blanc, mono- & autruche, héron, corbeau, paon, autour, \\
céros, tigre, castor, musqualiet, salamandre, & épervier, colombe, huppe, rossignol, cygne \\
lion, panthère, juments, éléphants, dragons, & (vi C) \\
couleuvre, anguilles, basilic, aspis, tygris & \\
(ii D), phénix, perroquet, pélican (ii F); & \\
echinus, dauphin, baleine, sirène (ii G) & \\
\hline
\end{tabular}

Tous ces animaux existaient dans l'imaginaire médiéval et ne représentaient pas, en effet, une grande nouveauté. Comme on peut le voir, les animaux fabuleux apparaissent surtout en Inde, ce qui est le cas dans la littérature didactique depuis

${ }^{13} \mathrm{Ce}$ texte se distingue des autres parce que l'auteur y inclut les connaissances zoologiques tirées du folklore, ce qui était rare dans les textes savants de l'époque médiévale.

${ }^{14}$ L'ouvrage anonyme La Lettre du Prêtre Jean, très diffusé en Europe entre le XII ${ }^{\mathrm{e}}$ et le XIII ${ }^{\mathrm{e}}$ siècles, décrivait les merveilles d'Inde. Sur la représentation de l'Inde dans la littérature didactique médiévale v. Marija Panić, « Le savoir sur l'Inde dans la littérature française des XII ${ }^{\mathrm{e}}$ et XIII" siècles », Nasledje n40, 2018, pp. 163-174. 
Ctésias, médecin grec qui a décrit l'Inde dans son ouvrage De Indica rédigé au V siècle avant Jésus-Christ. Ces mêmes animaux apparaissaient dans les ouvrages de Pline, de Solin, d'Isidore de Séville, de Jacques de Vitry et dans d'autres sources dont Gossouin de Metz s'est servi.

Les animaux connus figurent eux aussi dans l'Image du monde. On observe que les animaux familiers sont décrits en Europe et en Afrique ; toutefois, il s'agit de traits extraordinaires du comportement des animaux décrits qui n'ont pas toujours de rapport avec la réalité environnante. En effet, la zoologie médiévale n'avait pas le souci d'exactitude.

Il nous paraît opportun d'analyser la représentation d'un animal fabuleux et d'un animal réel : du basilic et du perroquet. « Illuec sont li basilique qui ont venimeus regart; et ocient les genz et les oiseaus et les bestes seulement de leur regart. Il a teste de coc et cors de serpant. [...] Il est rois de touz autres serpanz, ausi comme est li lyons seur les autres bestes » (ii F). Au Moyen Âge, le basilic était considéré comme le roi des reptiles ${ }^{15}$. Selon la littérature didactique médiévale, le lion était le roi des animaux terrestres, ou, selon certaines sources, le roi de tous les animaux ${ }^{16}$; l'aigle était le roi des oiseaux; le dauphin régnait sur les poissons ${ }^{17}$. Les auteurs des ouvrages didactiques médiévaux ne citaient pas tous les rois des animaux à la fois, ils se contentaient de parler du lion dans la plupart des $\operatorname{cas}^{18}$. Gossouin de Metz décrit le basilic comme un animal de l'Inde qui a un regard venimeux ; il tue les gens, les oiseaux et les bêtes rien que par son regard. Il a la tête du coq et le corps du serpent. Il est le roi des serpents, comme le lion est le roi des animaux.

Dans ce bref exemple, nous voyons que Gossouin de Metz montre la tendance à systématiser les connaissances : il énumère différentes catégories d'êtres vivants (hommes, oiseaux, bêtes) ; puis, il établit le lien entre deux rois des animaux, entre le lion et le basilic. Ceci n'était pas fréquent chez les auteurs médiévaux.

Li papegaut si sont cele part, qui sont tuit vert et reluisant comme paon, et ne sont pas plus grant d'un jai. Dont li plus gentill, ce dit on, ont en chascun des piez .v. doiz, et li vilain n'en ont que .iii. Si a la keue plus longue que n'a une pie, et a .i. bec courbé, et a grant langue et fournie. Qui l'a joene, il le puet faire parler as genz dedenz .ii. anz. (ii F)

L'autre exemple que nous citons est la description d'un animal réel, mais exotique. Il s'agit du perroquet, décrit avec force détails, et, ce qui est étonnant, de détails réalistes. Selon ce texte, là-bas - c'est-à-dire en Inde - il existe une

\footnotetext{
${ }^{15}$ M. Pastoureau, op. cit., 2011, p. 200.

${ }^{16}$ M. Pastoureau, op. cit., 2004, pp. 49-64.

${ }^{17}$ M. Pastoureau, op. cit., 2011, p. 179. Pour les naturalistes médiévaux, le dauphin était un poisson.

${ }^{18}$ À titre d'exemple, Emmanuel Walberg (éd.), Le Bestiaire de Philippe de Thaün, Lund, H. J. Möler / Paris, H. Welter, 1900, p. 2.
} 
sorte de perroquet dont le plumage vert et luisant ressemble à celui du paon ; il n'est pas plus grand qu'un geai ; sa queue est plus longue que celle d'une pie, et il a le bec courbé. Il existe deux sortes de perroquets : ceux qui ont trois doigts à leurs pieds, et ceux qui en ont cinq; ces derniers ont une meilleure réputation. Un jeune perroquet peut apprendre à parler aux humains dans l'espace de deux ans.

La description du perroquet nous paraît très réaliste. L'encyclopédiste messin a fait une synthèse réussie de plusieurs descriptions de cet oiseau exotique. Dans cette description de l'aspect physique, qui surprend par son exactitude, il est comparé au paon, au geai et à la pie, c'est-à-dire aux oiseaux qui faisaient partie de l'environnement européen médiéval, autant que du nôtre aujourd'hui. Gossouin de Metz instruit le lecteur sur deux différents types de perroquet et sur sa capacité d'apprendre à parler.

Il est évident qu'aucune interprétation symbolique ne fait partie de ses descriptions zoologiques. En fait, un trait significatif de la zoologie médiévale est complètement absent de ce texte. Il s'agit de la senefiance, du « sens caché », c'est-à-dire de l'interprétation symbolique explicite présente dans les textes sur les animaux, surtout dans les bestiaires. Les animaux dans l'Image du monde ne sont pas utilisés comme exemples qui peuvent être étudiés dans le but d'instruire le chrétien; aucune moralisation n'est présente dans les chapitres consacrés à la zoologie. L'ordre des chapitres dans l'Image du monde laisse imaginer la perfection divine ${ }^{19}$, de sorte qu'on ne saurait exclure la dimension symbolique de cet ouvrage ; reste quand même le fait que les animaux n'y sont pas utilisés comme points de départ pour une interprétation symbolique explicite.

On peut observer que la zoologie de Gossouin de Metz est encore très dépendante de ses sources (toujours citées par Prior dans les notes), qui ne sont d'ailleurs jamais soumises à un examen critique. Il n'y a pas non plus de véritables innovations de l'auteur. Dans ce patchwork d'informations tirées de sources différentes, l'auteur a réussi à composer un texte suivi qui se caractérise par une tentative de systématisation, dont les résultats sont encore très frustes. Toutefois, il est soulagé des lourdes explications étymologiques et de la moralisation qui étaient indissociables des descriptions zoologiques dans la littérature didactique du Moyen Âge.

\section{En guise de conclusion}

L'Image du monde de Gossouin de Metz est un exemple représentatif de l'état de la littérature didactique de son époque. D'un côté, cet ouvrage fait preuve de quelques innovations : le souci d'abolir les explications symboliques explicites, et de synthétiser en langue vulgaire les connaissances de son époque. Toutefois,

\footnotetext{
${ }^{19}$ Chantal Connochie-Bourgne, « Mise en récit et discours scientifique : les encyclopédies du XIII ${ }^{\mathrm{e}}$ siècle en langue vulgaire (Image du monde, Livres dou Tresor, Livre de Sidrac, Placides et Timéo) », dans Claude Thomasset (dir.), L'écriture du texte scientifique au Moyen Âge, Paris, Presses de l'Université Paris-Sorbonne, 2006, pp. 127-128.
} 
il reste très attaché à la tradition étant donné le caractère étonnant et pittoresque de ses descriptions des animaux exotiques, fantastiques ou familiers.

\section{SOURCES}

Baker, Craig Alexander (éd.), Le Bestiaire, version longue attribuée à Pierre de Beauvais, Paris, Honoré Champion, 2010.

Gosman, Martin (éd.) La Lettre du Prêtre Jean : les versions en ancien français et en ancien occitan, textes et commentaires, Groningen, Bouma's Boekhuis, 1982.

Ham, Edward B. (éd.), « The Cambrai Bestiary », Modern Philologyn³6/3, 1939, pp. 225-237.

Lindsay, Wallace Martin (éd.), Isidori Hispalensis Episcopi Etymologiarvm sive Originvm libri XX, New York / London, Oxford University Press, 2007-8.

Prior, Oscar H. (éd.), L'Image du monde du maître Gossouin : rédaction en prose, Lausanne, Librairie Payot et Cie, 1913.

Walberg, Emmanuel (éd.), Le Bestiaire de Philippe de Thaün, Lund, H. J. Möler / Paris, H. Welter, 1900.

\section{LITTERATURE}

Connochie, Chantal, « Gossuin de Metz », dans Claude Gauvard, Alain de Libera et Michel Zink (dir.), Dictionnaire du Moyen Âge, Paris, PUF, 2002, p. 597.

Connochie-Bourgne, Chantal, «Mise en récit et discours scientifique : les encyclopédies du XIII ${ }^{\mathrm{e}}$ siècle en langue vulgaire (Image du monde, Livres dou Tresor, Livre de Sidrac, Placides et Timéo) », dans Claude Thomasset (dir.), L'écriture du texte scientifique au Moyen Âge, Paris, Presses de l'Université Paris-Sorbonne, 2006, pp. 117-131.

Delort, Robert, Les animaux ont une histoire, Paris, Seuil, 1984.

Dragojlović, Dragoljub, Fiziolog u Srba (thèse de doctorat), Faculté de philosophie, Université de Belgrade, Univerzitetska biblioteka à Belgrade, cote RD 3451, 1968.

Duval, Frédéric, « Les traductions françaises d'Isidore de Séville au Moyen Âge », Cahiers de recherches médiévales et humanistes n¹6, 2008, pp. 93-105.

Eko, Umberto, Umetnost i lepo u estetici srednjeg veka (prevele Tanja Majstorović i Snežana Brajović), Novi Sad, Svetovi / Beograd, Kultura, 1992.

Gregory, Tullio, « Nature », dans Jacques Le Goff, Jean-Claude Schmitt et Jean Alessio (éd.), Dictionnaire raisonné de l'Occident médiéval, Paris, Fayard, 1999, pp. 806-819. 
Muratova, Xénia, « Adam donne leurs noms aux animaux. L'iconographie de la scène dans l'art du Moyen Âge : les manuscrits des bestiaires enluminés du XII ${ }^{\mathrm{e}}$ et du XIII ${ }^{\mathrm{e}}$ siècle » Studi medievali Firenze n¹8/2, 1977, pp. 367-394.

Панић, Марија, „Један вид средњовековне зооморфне симболике: приказ и тумачење природе у француским бестијарима", Зборник Матице српске за књижевност и језик $\mathrm{n}^{\circ} 63 / \mathrm{III}, 2015$, стр. 695-718.

Panić, Marija, « Le savoir sur l’Inde dans la littérature française des XII ${ }^{e}$ et XIII ${ }^{\mathrm{e}}$ siècles », Nasledje $\mathrm{n}^{\circ} 40,2018$, pp. 163-174.

Pastoureau, Michel, Une histoire symbolique du Moyen Âge occidental, Paris, Seuil, 2004.

Pastoureau, Michel, Bestiaires du Moyen Âge, Paris, Seuil, 2011.

Strubel, Armand, 'Grant sénéfiance a': allégorie et littérature au Moyen Âge, Paris, Honoré Champion, 2002.

Замбон, Франческо, „Теологија бестијаријума” (превела Александра Манчић Милић), Градай n75-78, 1986-1987, стр. 121-134.

Марија Панић

\title{
ЗООЛОГИЈА У СЛИЦИ СВЕТА ГОСУЕНА ИЗ МЕЦА \\ (Резиме)
}

\begin{abstract}
У чланку се разматра приказ животињског царства у енциклопедији Слика света (Image $d u$ monde) Госуена из Меца (средина тринаестог века), прве суме знања на вулгарном језику. Наводимо изворе које је користио енциклопедиста из Меца (античке и средњовековне латинске ауторе), потом класификацију животиња и њихов приказ у тексту. На основу поређења са другим дидактичким (зоолошким и космографским) текстовима из његовог времена, закључујемо да је аутор, у складу са тенденцијама тога времена, умањио симболичку димензију свога дела.
\end{abstract}

Кључне речи: Госуен из Меца, Слика света, животиња, симболизам, средњовековни енциклопедизам.

Примљено 23. јула 2020, прихваћено за објављивање 10. децембра 2020. године. 